\title{
Emergence of a Complete Heavy-Quark Spin Symmetry Multiplet: Seven Molecular Pentaquarks in Light of the Latest LHCb Analysis
}

\author{
Ming-Zhu Liu, ${ }^{1}$ Ya-Wen Pan, ${ }^{1}$ Fang-Zheng Peng, ${ }^{1}$ Mario Sánchez Sánchez, ${ }^{2}$ Li-Sheng Geng, ${ }^{1,3, *}$ \\ Atsushi Hosaka, ${ }^{4,5, \dagger}$ and Manuel Pavon Valderrama ${ }^{1,6, \$}$ \\ ${ }^{1}$ School of Physics and Nuclear Energy Engineering, Beihang University, Beijing 100191, China \\ ${ }^{2}$ Centre d'Études Nucléaires, CNRS/IN2P3, Université de Bordeaux, 33175 Gradignan, France \\ ${ }^{3}$ Beijing Key Laboratory of Advanced Nuclear Materials and Physics and Beijing Advanced Innovation Center \\ for Big Data-based Precision Medicine, Beihang University, Beijing 100191,China \\ ${ }^{4}$ Research Center for Nuclear Physics (RCNP), Osaka University, Ibaraki 567-0047, Japan \\ ${ }^{5}$ Advanced Science Research Center, Japan Atomic Energy Agency (JAEA), Tokai 319-1195, Japan \\ ${ }^{6}$ International Research Center for Nuclei and Particles in the Cosmos and Beijing Key Laboratory \\ of Advanced Nuclear Materials and Physics, Beihang University, Beijing 100191, China
}

(Received 12 April 2019; revised manuscript received 9 May 2019; published 21 June 2019)

\begin{abstract}
A recent analysis by the LHCb Collaboration suggests the existence of three narrow pentaquarklike states - the $P_{c}(4312), P_{c}(4440)$, and $P_{c}(4457)$-instead of just one in the previous analysis [the $\left.P_{c}(4450)\right]$. The closeness of the $P_{c}(4312)$ to the $\bar{D} \Sigma_{c}$ threshold and the $P_{c}(4440)$ and $P_{c}(4457)$ to the $\bar{D}^{*} \Sigma_{c}$ threshold suggests a molecular interpretation of these resonances. We show that these three pentaquarklike resonances can be naturally accommodated in a contact-range effective field theory description that incorporates heavy-quark spin symmetry. This description leads to the prediction of all the seven possible $S$-wave heavy antimeson-baryon molecules [that is, there should be four additional molecular pentaquarks in addition to the $P_{c}(4312), P_{c}(4440)$, and $\left.P_{c}(4457)\right]$, providing the first example of a heavy-quark spin symmetry molecular multiplet that is complete. If this is confirmed, it will not only give us an impressive example of the application of heavy-quark symmetries and effective field theories in hadron physics, it will also uncover a clear and powerful ordering principle for the molecular spectrum, reminiscent of the SU(3)-flavor multiplets to which the light hadron spectrum conforms.
\end{abstract}

DOI: $10.1103 /$ PhysRevLett.122.242001

In 2015 the LHCb Collaboration discovered the existence of two pentaquarklike resonances, which are usually referred to as $P_{c}(4380)$ and $P_{c}(4450)$ due to their masses [1]. This experimental discovery triggered intense theoretical speculations on the nature of these states, their decays, and production mechanisms. In particular, the closeness of the $P_{c}(4450)$ to a few meson-baryon thresholds $\left[\bar{D}^{*} \Sigma_{c}\right.$, $\bar{D}^{*} \Sigma_{c}^{*}, \bar{D} \Lambda_{c}(2595)$, and $\left.\chi_{c 1} p\right]$ leads naturally to the conjecture that it is a meson-baryon bound state (a conjecture further cemented by a series of theoretical predictions that predated its observation [2-8]), with the most popular explanations being a $\bar{D}^{*} \Sigma_{c}[9-11]$ or a $\bar{D}^{*} \Sigma_{c}^{*}$ molecule $[12,13]$ [in these two cases in the isospin $I=\frac{1}{2}$ configuration and probably with a small admixture of $\bar{D} \Lambda_{c}(2595)$ $[14,15]]$, and a $\chi_{c 1} p$ molecule [16]. There are also nonmolecular explanations for this state, which include that it

Published by the American Physical Society under the terms of the Creative Commons Attribution 4.0 International license. Further distribution of this work must maintain attribution to the author(s) and the published article's title, journal citation, and DOI. Funded by SCOAP ${ }^{3}$. might be a genuine pentaquark [17-23], that threshold effects might play a role [24,25] (see also Ref. [26] for a detailed discussion), baryocharmonia [27], a molecule bound by color chemistry [28], and a soliton [29].

The original analysis of Ref. [1] has been recently updated by the LHCb Collaboration in Ref. [30], where it has been found that the previous $P_{c}(4450)$ actually contains two peaks - the $P_{c}(4440)$ and $P_{c}(4457)$ - and that there is a third narrow peak, the $P_{c}(4312)$. Their masses and widths are

$$
\begin{array}{ll}
m_{P_{c 1}}=4311.9 \pm 0.7_{-0.6}^{+6.8}, & \Gamma_{P_{c 1}}=9.8 \pm 2.7_{-4.5}^{+3.7}, \\
m_{P_{c 2}}=4440.3 \pm 1.3_{-4.7}^{+4.1}, & \Gamma_{P_{c 2}}=20.6 \pm 4.9_{-10.1}^{+8.7}, \\
m_{P_{c 3}}=4457.3 \pm 0.6_{-1.7}^{+4.1}, & \Gamma_{P_{c 3}}=6.4 \pm 2.0_{-1.9}^{+5.7},
\end{array}
$$

all in units of $\mathrm{MeV}$ and for which we have used the notation $P_{c 1}, P_{c 2}$, and $P_{c 3}$ to refer to the three states $P_{c}(4312)$, $P_{c}(4440)$, and $P_{c}(4457)$ (that is, we have ordered them according to their masses). It is interesting to notice that the mass of the previous $P_{c}(4450)$ roughly coincides with the geometric mean of the masses of the new $P_{c}(4440)$ and $P_{c}(4457)$. The $P_{c}(4312)$ pentaquarklike state is near to the 
$\bar{D} \Sigma_{c}$ threshold, while the other two are close to the $\bar{D}^{*} \Sigma_{c}$ one. When translated into binding energies we obtain $B_{1}=8.9, B_{2}=21.8$, and $B_{3}=4.8 \mathrm{MeV}$ for the $P_{c}(4312)$, $P_{c}(4440)$, and $P_{c}(4457)$, respectively. Of course this closeness to threshold has already been noted by theoreticians in Refs. [31-33]. If these findings are confirmed it will not only strongly support the molecular hypothesis, but it will also provide us with the most impressive illustration of the application of heavy-quark spin symmetry (HQSS) [34-37] to hadronic molecules so far. In particular, this experimental analysis will result in the prediction of the first full HQSS molecular multiplet of the hidden-charm molecular pentaquarks.

Heavy-hadron molecules, i.e., bound states that include one or more heavy hadrons, were conjectured decades ago $[38,39]$. Owing to the combination of light- and heavyquark content, heavy-hadron molecules have a high degree of symmetry which can be exploited to determine their spectrum [40-49]. HQSS manifests in the existence of interesting patterns in the heavy-molecular spectrum. The most evident of these patterns applies to the $Z_{c}$ 's and $Z_{b}$ 's twin resonances discovered by BESIII [50-53] and Belle $[54,55]$, respectively. If they are bound states of a heavy meson and antimeson, being either a charm meson or bottom meson, HQSS predicts that the $S$-wave potential in the $J^{P C}=1^{+-}$channel is $[42,43]$

$$
V\left(1^{+-}, P^{*} \bar{P}\right)=V\left(1^{+-}, P^{*} \bar{P}^{*}\right),
$$

independently of the particle content, where $P=D, \bar{B}$ and $P^{*}=D^{*}, \bar{B}^{*}$. This specific pattern indeed explains why the $Z_{c}$ 's and $Z_{b}$ 's appear in pairs, both of which are at similar distances from the $P^{*} P$ and $P^{*} P^{*}$ open heavy flavor thresholds. A similar pattern applies to the $1^{++}$and $2^{++}$ heavy meson-antimeson interaction [44-46]:

$$
V\left(1^{++}, P^{*} \bar{P}\right)=V\left(2^{++}, P^{*} \bar{P}^{*}\right) .
$$

If we assume the $X(3872)$ resonance to be a $D^{*} \bar{D}$ bound state with $J^{\mathrm{PC}}=1^{++}$, this symmetry relation suggests the existence of a $2^{++} D^{*} \bar{D}^{*}$ partner with a mass of $4012 \mathrm{MeV}$. However, the location of the $X(3872)$ overlaps with the $D^{0 *} \bar{D}^{0}$ threshold within experimental errors, which implies that the existence of the $2^{++}$partner of the $X$ is not guaranteed if we take into account this error source (besides, there are other uncertainties [56,57]); see Ref. [58] for a more complete discussion. At this point we notice that there are six possible heavy meson-antimeson molecules, forming a HQSS multiplet that can accommodate up to six resonances. However, the known heavy meson-antimeson molecules are all too close to threshold, indicating that most probably this multiplet structure is unlikely to be fully realized in nature, leaving us with an incomplete pattern.
In this Letter we argue that the new LHCb results [30] imply that the heavy antimeson-baryon molecules will probably provide the first example of a full and intact HQSS molecular multiplet. For this we begin by explaining the constraints that HQSS imposes on the $S$-wave heavy antimeson-baryon interaction, as has been recently derived in Ref. [59]. HQSS implies that we can describe the seven $S$-wave $\bar{D}^{(*)} \Sigma_{c}^{(*)}$ molecules with two coupling constants. If we additionally assume that the heavy antimeson-baryon molecules can be described in terms of a contact-range effective field theory, the potential for the $\bar{D} \Sigma_{c}, \bar{D} \Sigma_{c}^{*}, \bar{D}^{*} \Sigma_{c}$, and $\bar{D}^{*} \Sigma_{c}^{*}$ molecules is [59]

$$
\begin{gathered}
V\left(\frac{1^{-}}{2}, \bar{D} \Sigma_{c}\right)=C_{a}, \\
V\left(\frac{3}{2}^{-}, \bar{D} \Sigma_{c}^{*}\right)=C_{a}, \\
V\left(\frac{1^{-}}{2}, \bar{D}^{*} \Sigma_{c}\right)=C_{a}-\frac{4}{3} C_{b}, \\
V\left(\frac{3^{-}}{2}, \bar{D}^{*} \Sigma_{c}\right)=C_{a}+\frac{2}{3} C_{b}, \\
V\left(\frac{1^{-}}{2}, \bar{D}^{*} \Sigma_{c}^{*}\right)=C_{a}-\frac{5}{3} C_{b}, \\
V\left(\frac{3^{-}}{2}, \bar{D}^{*} \Sigma_{c}^{*}\right)=C_{a}-\frac{2}{3} C_{b}, \\
V\left(\frac{5^{-}}{2}, \bar{D}^{*} \Sigma_{c}^{*}\right)=C_{a}+C_{b},
\end{gathered}
$$

with $C_{a}$ and $C_{b}$ unknown coupling constants. This potential is renormalized by including a separable regulator and a cutoff $\Lambda$ in momentum space and allowing the couplings to depend on this cutoff,

$$
\left\langle p\left|V_{\Lambda}\right| p^{\prime}\right\rangle=C(\Lambda) f\left(\frac{p}{\Lambda}\right) f\left(\frac{p^{\prime}}{\Lambda}\right)
$$

where $p, p^{\prime}$ are the initial and final center-of-mass momenta of the two-body system and $C$ represents the linear combination of $C_{a}$ and $C_{b}$ corresponding to the heavy antimeson-baryon molecule under consideration; see Eqs. (3)-(9) for details. For the regulator we choose a Gaussian one, $f(x)=e^{-x^{2}}$, while for the cutoff we consider the range $\Lambda=0.5-1.0 \mathrm{GeV}$, where we notice that if the problem has been properly renormalized, the dependence of the predictions on the cutoff will be small. The potential is then included in a dynamical equation, e.g., LippmannSchwinger: 


$$
\phi(k)+\int \frac{d^{3} p}{(2 \pi)^{3}}\left\langle k\left|V_{\Lambda}\right| p\right\rangle \frac{\phi(p)}{B_{2}+\frac{p^{2}}{2 \mu}}=0,
$$

with $\phi$ the vertex function, $\mu$ the reduced mass of the system, and $B_{2}$ the binding energy, where solutions of this dynamical equation correspond to bound states. Alternatively, with the purpose of checking regulator independence, we can use a delta-shell regulator in coordinate space,

$$
V\left(r ; R_{c}\right)=C\left(R_{c}\right) \frac{\delta\left(r-R_{c}\right)}{4 \pi R_{c}^{2}},
$$

with a cutoff in the range $R_{c}=0.5-1.0 \mathrm{fm}$, i.e., of the order of the typical hadron size, and solve the $S$-wave Schrödinger equation. The delta-shell regulator is convenient-it allows for analytic results-but we stress that any other choice of regulator will work too. We will not show detailed results for the delta-shell or other regulators here, but simply comment that the differences with the Gaussian regulator are minor.

We notice that both the $P_{c}(4440)$ and $P_{c}(4457)$ are good $\bar{D}^{*} \Sigma_{c}$ molecular candidates, but their $J^{P}$ is not known. The natural expectation in hadron physics is that states with higher spin will have a higher mass, but hadronic molecules might deviate from this trend depending on the binding mechanism (e.g., the spectrum predicted in Ref. [6] for the molecular pentaquarks). Here we will not make a priori assumptions and will let the comparison between theory and experiment decide instead. Thus we distinguish two scenarios, $\mathrm{A}$ and $\mathrm{B}$, where scenario $\mathrm{A}$ corresponds to assuming that the $P_{c}(4440)$ and $P_{c}(4457)$ are $J^{P}=(1 / 2)^{-}$ and $J^{P}=(3 / 2)^{-}$, respectively, while scenario B corresponds to the opposite identification. Each of these choices completely fixes the effective field theory potential and allows us to predict the location of the $J^{P}=(1 / 2)^{-} \bar{D} \Sigma_{c}$ molecule, which in scenario A we predict at

$$
M_{A}\left(\bar{D} \Sigma_{c}\right)=(4311.8-4313.0) \mathrm{MeV},
$$

which is extremely close to the experimental value, $M_{P_{c 1}}=4311.9 \mathrm{MeV}$, and where the range corresponds to the cutoff variation $\Lambda=0.5-1.0 \mathrm{GeV}$. In contrast to this, in scenario B the $P_{c}(4312)$ resonance is predicted at

$$
M_{B}\left(\bar{D} \Sigma_{c}\right)=(4306.3-4307.7) \mathrm{MeV},
$$

which is not too close to the expected location of the $P_{c}(4312)$ resonance but still compatible within the experimental errors. The predictions are fairly independent not only on the cutoff, but also on the choice of regulator: had we used the delta-shell regulator of Eq. (12) instead of the Gaussian regulator of Eq. (10), the predictions would have been

$$
\begin{aligned}
M_{A}^{\prime}\left(\bar{D} \Sigma_{c}\right) & =(4312.1-4313.1) \mathrm{MeV}, \\
M_{B}^{\prime}\left(\bar{D} \Sigma_{c}\right) & =(4306.7-4308.0) \mathrm{MeV},
\end{aligned}
$$

for the $R_{c}=0.5-1.0 \mathrm{fm}$ cutoff range, which indicates a preference for scenario A. This is also the case for other regulators, e.g., a square-well or a Gaussian potential in coordinate space. However, note that we have not propagated the uncertainty in the masses of the input data-the $P_{c}(4440)$ and $P_{c}(4457)$ - nor have we taken into account the experimental uncertainty in the location of the $P_{c}(4312)$. That is, the preference for scenario A is probably not particularly strong. For comparison purposes, the seminal works of Refs. [2-5] predict the $J^{P}=(1 / 2)^{-}$ and $(3 / 2)^{-} \bar{D}^{*} \Sigma_{c}$ states to be degenerate. The molecular identifications of the $P_{c}(4440)$ and $P_{c}(4457)$ that are derived from the one boson exchange models of Refs. [32,60] are equivalent to our scenario A, i.e., the scenario favored by our calculations. On the other hand, Ref. [6] predicts the $(3 / 2)^{-} \bar{D}^{*} \Sigma_{c}$ molecule to be more bound, a conclusion which is deduced from the strength and sign of the one pion exchange potential in $S$ waves and which corresponds to our scenario B.

Yet, as previously explained, the really exciting aspect of being able to determine both $C_{a}$ and $C_{b}$ is that now we can predict all seven heavy antimeson-baryon molecules. This is done in Table I for scenarios A and B, where in both cases the seven molecules are always predicted but the specifics of their location changes slightly depending on the

TABLE I. Predictions for the $S$-wave HQSS molecular multiplet of heavy antimeson-baryon molecules, as derived from the lowest-order contact-range potential which contains two unknown couplings $C_{a}$ and $C_{b}$. The potential for each particle and spin channel (the "Molecule" and " $J$ " columns) can be checked in Eqs. (3)-(9). In all cases we assume that the isospin of the listed molecules is $I=\frac{1}{2}$. We determine the value of the $C_{a}$ and $C_{b}$ couplings from the condition of reproducing the location of the $P_{c}(4440)$ and $P_{c}(4457)$ resonances, which are known to be close to the $\bar{D}^{*} \Sigma_{c}$ threshold. We do not know, however, the quantum numbers of the $P_{c}(4440)$ and $P_{c}(4457)$, but consider two possibilities instead: in scenario A the $(1 / 2)^{-}$molecule is identified with the $P_{c}(4440)$ and the $(3 / 2)^{-}$with the $P_{c}(4457)$, while scenario B assumes the opposite identification.

\begin{tabular}{lcccc}
\hline \hline Scenario & Molecule & $J^{P}$ & $B(\mathrm{MeV})$ & $M(\mathrm{MeV})$ \\
\hline A & $\bar{D} \Sigma_{c}$ & $(1 / 2)^{-}$ & $7.8-9.0$ & $4311.8-4313.0$ \\
A & $\bar{D} \Sigma_{c}^{*}$ & $(3 / 2)^{-}$ & $8.3-9.2$ & $4376.1-4377.0$ \\
A & $\bar{D}^{*} \Sigma_{c}$ & $(1 / 2)^{-}$ & Input & 4440.3 \\
A & $\bar{D}^{*} \Sigma_{c}$ & $(3 / 2)^{-}$ & Input & 4457.3 \\
A & $\bar{D}^{*} \Sigma_{c}^{*}$ & $(1 / 2)^{-}$ & $25.7-26.5$ & $4500.2-4501.0$ \\
A & $\bar{D}^{*} \Sigma_{c}^{*}$ & $(3 / 2)^{-}$ & $15.9-16.1$ & $4510.6-4510.8$ \\
A & $\bar{D}^{*} \Sigma_{c}^{*}$ & $(5 / 2)^{-}$ & $3.2-3.5$ & $4523.3-4523.6$ \\
B & $\bar{D} \Sigma_{c}$ & $(1 / 2)^{-}$ & $13.1-14.5$ & $4306.3-4307.7$ \\
B & $\bar{D} \Sigma_{c}^{*}$ & $(3 / 2)^{-}$ & $13.6-14.8$ & $4370.5-4371.7$ \\
B & $\bar{D}^{*} \Sigma_{c}$ & $(1 / 2)^{-}$ & Input & 4457.3 \\
B & $\bar{D}^{*} \Sigma_{c}$ & $(3 / 2)^{-}$ & Input & 4440.3 \\
B & $\bar{D}^{*} \Sigma_{c}^{*}$ & $(1 / 2)^{-}$ & $3.1-3.5$ & $4523.2-4523.6$ \\
B & $\bar{D}^{*} \Sigma_{c}^{*}$ & $(3 / 2)^{-}$ & $10.1-10.2$ & $4516.5-4516.6$ \\
B & $\bar{D}^{*} \Sigma_{c}^{*}$ & $(5 / 2)^{-}$ & $25.7-26.5$ & $4500.2-4501.0$ \\
\hline \hline
\end{tabular}


chosen scenario, particularly in regards to the $\bar{D}^{*} \Sigma_{c}^{*}$ molecules: for scenario A binding decreases with the spin quantum number, while the contrary is true for scenario B. We notice the prediction of a $\bar{D} \Sigma_{c}^{*}$ bound state at 4370 $4380 \mathrm{MeV}$, though the identification with the $P_{c}(4380)$ pentaquark peak of Ref. [1] is problematic owing to the broad nature of this state. Our conclusion that the HQSS multiplet for the heavy meson-baryon molecules is complete has been independently confirmed in Ref. [61].

To summarize, the recent analysis of the $\mathrm{LHCb}$ Collaboration supports the hypothesis that the pentaquarklike $P_{c}(4312), P_{c}(4440)$, and $P_{c}(4457)$ resonances are indeed $\bar{D} \Sigma_{c}$ and $\bar{D}^{*} \Sigma_{c}$ molecules. In addition, this experimental observation unlocks the possibility of the theoretical prediction of all seven $S$-wave heavy antimeson-baryon molecules, which incidentally provides the first example of a full and complete HQSS multiplet for hadronic molecules. The identification of the $P_{c}(4440)$ and $P_{c}(4457)$ with $\bar{D}^{*} \Sigma_{c}$ bound states is ambiguous: both the $J^{P}=$ $(1 / 2)^{-}$and $J^{P}=(3 / 2)^{-}$quantum numbers are in principle possible. Even though the specific spin of the $P_{c}(4440)$ and $P_{c}(4457)$ molecular candidates is inconsequential for the prediction of the HQSS multiplet, the spectroscopic predictions of the contact-range effective field theory we use in this Letter indicate a preference for identifying the $P_{c}(4440)$ and $P_{c}(4457)$ with the $J^{P}=(1 / 2)^{-}$and the $J^{P}=(3 / 2)^{-} \bar{D}^{*} \Sigma_{c}$ molecules, respectively. Though the present theoretical exploration focuses only on the spectroscopy of the molecular pentaquarks, the eventual discovery of the missing members of the HQSS multiplets at their predicted locations will by itself represent a very strong case in favor of their molecular nature. Yet future investigation of their decays and production mechanisms will be essential to disentangle the nature of these pentaquarklike states. Finally, we stress that the idea of HQSS multiplets provides a clear and concise ordering principle for molecular states which, in analogy to the SU(3)-flavor multiplets in the light-hadron sector, has the potential to help to interpret the results of future experimental searches of exotic states and to improve our understanding of the nonperturbative strong interaction.

This work is partly supported by the National Natural Science Foundation of China under Grant No. 11735003, the Fundamental Research Funds for the Central Universities, and the Thousand Talents Plan for Young Professionals. A. H. acknowledges support from Grants-inAid for Scientific Research (No. JP17K05441 (C)) and for Scientific Research on Innovative Areas (No. 18H05407).

\footnotetext{
*lisheng.geng@buaa.edu.cn

†hosaka@rcnp.osaka-u.ac.jp

*mpavon@buaa.edu.cn
}

[1] R. Aaij et al. (LHCb Collaboration), Phys. Rev. Lett. 115, 072001 (2015).

[2] J.-J. Wu, R. Molina, E. Oset, and B. S. Zou, Phys. Rev. Lett. 105, 232001 (2010).

[3] J.-J. Wu, R. Molina, E. Oset, and B. S. Zou, Phys. Rev. C 84, 015202 (2011).

[4] J.-J. Wu and B. S. Zou, Phys. Lett. B 709, 70 (2012).

[5] C. W. Xiao, J. Nieves, and E. Oset, Phys. Rev. D 88, 056012 (2013).

[6] M. Karliner and J. L. Rosner, Phys. Rev. Lett. 115, 122001 (2015).

[7] W. L. Wang, F. Huang, Z. Y. Zhang, and B. S. Zou, Phys. Rev. C 84, 015203 (2011).

[8] Z.-C. Yang, Z.-F. Sun, J. He, X. Liu, and S.-L. Zhu, Chin. Phys. C 36, 6 (2012).

[9] L. Roca, J. Nieves, and E. Oset, Phys. Rev. D 92, 094003 (2015).

[10] J. He, Phys. Lett. B 753, 547 (2016).

[11] C. W. Xiao and U. G. Meißner, Phys. Rev. D 92, 114002 (2015).

[12] R. Chen, X. Liu, X.-Q. Li, and S.-L. Zhu, Phys. Rev. Lett. 115, 132002 (2015)

[13] H.-X. Chen, W. Chen, X. Liu, T. G. Steele, and S.-L. Zhu, Phys. Rev. Lett. 115, 172001 (2015).

[14] T. J. Burns, Eur. Phys. J. A 51, 152 (2015).

[15] L. S. Geng, J. X. Lu, and M. P. Valderrama, Phys. Rev. D 97, 094036 (2018).

[16] U.-G. Meißner and J. A. Oller, Phys. Lett. B 751, 59 (2015).

[17] D. Diakonov, V. Petrov, and M. V. Polyakov, Z. Phys. A 359, 305 (1997).

[18] R. L. Jaffe and F. Wilczek, Phys. Rev. Lett. 91, 232003 (2003).

[19] S. G. Yuan, K. W. Wei, J. He, H. S. Xu, and B. S. Zou, Eur. Phys. J. A 48, 61 (2012).

[20] L. Maiani, A. D. Polosa, and V. Riquer, Phys. Lett. B 749, 289 (2015).

[21] R. F. Lebed, Phys. Lett. B 749, 454 (2015).

[22] G.-N. Li, X.-G. He, and M. He, J. High Energy Phys. 12 (2015) 128.

[23] Z.-G. Wang, Eur. Phys. J. C 76, 70 (2016).

[24] F.-K. Guo, Ulf-G. Meißner, W. Wang, and Z. Yang, Phys. Rev. D 92, 071502(R) (2015).

[25] X.-H. Liu, Q. Wang, and Q. Zhao, Phys. Lett. B 757, 231 (2016).

[26] M. Bayar, F. Aceti, F.-K. Guo, and E. Oset, Phys. Rev. D 94, 074039 (2016).

[27] V. Kubarovsky and M. B. Voloshin, Phys. Rev. D 92, 031502(R) (2015).

[28] A. Mironov and A. Morozov, Pis'ma Zh. Eksp. Teor. Fiz. 102, 302 (2015) [JETP Lett. 102, 271 (2015)].

[29] N. N. Scoccola, D. O. Riska, and M. Rho, Phys. Rev. D 92 , 051501(R) (2015).

[30] R. Aaij et al. (LHCb Collaboration), Phys. Rev. Lett. 122, 222001 (2019).

[31] H.-X. Chen, W. Chen, and S.-L. Zhu, arXiv:1903.11001.

[32] R. Chen, X. Liu, Z.-F. Sun, and S.-L. Zhu, arXiv: 1903.11013.

[33] Z.-H. Guo and J. A. Oller, Phys. Lett. B 793, 144 (2019).

[34] N. Isgur and M. B. Wise, Phys. Lett. B 232, 113 (1989).

[35] N. Isgur and M. B. Wise, Phys. Lett. B 237, 527 (1990). 
[36] M. Neubert, Phys. Rep. 245, 259 (1994).

[37] A. V. Manohar and M. B. Wise, Cambridge Monogr. Part. Phys., Nucl. Phys., Cosmol. 10, 1 (2000).

[38] M. Voloshin and L. Okun, JETP Lett. 23, 333 (1976).

[39] A. De Rujula, H. Georgi, and S. Glashow, Phys. Rev. Lett. 38, 317 (1977).

[40] M. T. AlFiky, F. Gabbiani, and A. A. Petrov, Phys. Lett. B 640, 238 (2006).

[41] F.-K. Guo, C. Hanhart, and Ulf-G. Meissner, Phys. Rev. Lett. 102, 242004 (2009).

[42] M. B. Voloshin, Phys. Rev. D 84, 031502(R) (2011).

[43] T. Mehen and J.W. Powell, Phys. Rev. D 84, 114013 (2011).

[44] M. Pavón Valderrama, Phys. Rev. D 85, 114037 (2012).

[45] J. Nieves and M. Pavón Valderrama, Phys. Rev. D 86, 056004 (2012).

[46] C. Hidalgo-Duque, J. Nieves, and M. P. Valderrama, Phys. Rev. D 87, 076006 (2013).

[47] F.-K. Guo, C. Hidalgo-Duque, J. Nieves, and M. Pavón Valderrama, Phys. Rev. D 88, 054007 (2013).

[48] F.-K. Guo, C. Hidalgo-Duque, J. Nieves, and M.P. Valderrama, Phys. Rev. D 88, 054014 (2013).

[49] J.-X. Lu, L.-S. Geng, and M. P. Valderrama, Phys. Rev. D 99, 074026 (2019).
[50] M. Ablikim et al. (BESIII Collaboration), Phys. Rev. Lett. 110, 252001 (2013).

[51] Z. Q. Liu et al. (Belle Collaboration), Phys. Rev. Lett. 110, 252002 (2013).

[52] M. Ablikim et al. (BESIII Collaboration), Phys. Rev. Lett. 111, 242001 (2013).

[53] M. Ablikim et al. (BESIII Collaboration), Phys. Rev. Lett. 113, 212002 (2014).

[54] A. Bondar et al. (Belle Collaboration), Phys. Rev. Lett. 108, 122001 (2012).

[55] A. Garmash et al. (Belle Collaboration), Phys. Rev. D 91, 072003 (2015).

[56] E. Cincioglu, J. Nieves, A. Ozpineci, and A. U. Yilmazer, Eur. Phys. J. C 76, 576 (2016).

[57] V. Baru, E. Epelbaum, A. A. Filin, C. Hanhart, U.-G. Meißner, and A. V. Nefediev, Phys. Lett. B 763, 20 (2016).

[58] M.-Z. Liu, T.-W. Wu, M. P. Valderrama, J.-J. Xie, and L.-S. Geng, Phys. Rev. D 99, 094018 (2019).

[59] M.-Z. Liu, F.-Z. Peng, M. Sánchez Sánchez, and M. Pavon Valderrama, Phys. Rev. D 98, 114030 (2018).

[60] J. He, Eur. Phys. J. C 79, 393 (2019).

[61] C. W. Xiao, J. Nieves, and E. Oset, arXiv:1904.01296. 